\title{
Liderazgo y comportamiento innovador del trabajador en personal administrativo de una institución educativa*
}

\author{
Leadership and Innovative Work Behavior in the \\ Administrative Staff of an Educational Institution
}

\author{
Maycoll Prieto Antolines \\ ORCID 0000-0003-3146-6930 \\ Francoise Contreras \\ ORCID 0000-0002-2627-0813 \\ Juan C. Espinosa \\ ORCID 0000-0002-1643-2233 \\ Universidad del Rosario, Colombia
}

Recibido: 22 de junio de 2019

Revisado: 7 de agosto de 2019

Aceptado: 2 de noviembre 2019

\section{Resumen}

El propósito de este estudio fue describir las prácticas de liderazgo que percibe un grupo de 145 empleados respecto a su jefe inmediato y estimar si éstas guardan relación con su comportamiento innovador en el trabajo. El estudio se realizó en una institución de educación superior con funcionarios del área administrativa que pertenecían al mismo departamento. Para esto, se utilizó la Escala de Liderazgo para la Innovación (Innovational Leadership Scale [LL-15]) de Contreras, Espinosa y Dornberger (2019) y la Escala de Comportamiento Innovador en el Trabajo de Janssen (2000). Ambos cuestionarios mostraron altos niveles de confiabilidad (coeficientes alfa de Cronbach superiores a .90). De acuerdo con los resultados, se observa un nivel medio de desarrollo en las dos variables estudiadas. Así mismo, se observó que los empleados con mayor comportamiento innovador perciben un mayor liderazgo para la innovación en sus jefes. Estos hallazgos contribuyen a la formación de líderes que fomenten la innovación en

Artículo de investigación. Citar como: Prieto, M. G., Contreras, F., \& Espinosa, J. C. (2020). Liderazgo para la innovación y comportamiento innovador en un grupo de trabajadores administrativos de una universidad privada. Diversitas: Perspectivas en Psicología, 16(1), 25-35. Dol: https: / /doi.org/10.15332/22563067.5540

Autor de correspondencia: Francoise Contreras, Universidad del Rosario, Escuela de Administración. Dirección postal: Autopista Norte, Calle 200, Universidad del Rosario, Sede del Emprendimiento y la Innovación. Correo electrónico: francoise.contreras@urosario.edu.co 
las organizaciones y muestran la necesidad de continuar estudiando este estilo de liderazgo en las empresas colombianas en relación con el comportamiento innovador de sus trabajadores a fin de contribuir al desarrollo de organizaciones más adaptables a las circunstancias cambiantes del entorno.

Palabras clave: liderazgo, innovación, comportamiento innovador del trabajador, Liderazgo para la innovación.

\section{Abstract}

The purpose of this study was to describe the leadership practices perceived by a group of $145 \mathrm{em}$ ployees with regard to their immediate boss and to estimate whether these practices are related to their innovative behavior at work. The study was conducted in a higher education institution with employees from the administrative area who belonged to the same department. To this end, the Innovational Leadership Scale (ILS-15]) of Contreras, Espinosa, and Dornberger (2019) and the Janssen Innovative Work Behaviour Scale (2000) were used. Both questionnaires showed high levels of reliability (Cronbach's alpha coefficients greater than .90). According to the results, an average level of development may observed in the two variables that were studied. Likewise, it was observed that the employees with greater innovative behavior perceive greater leadership for innovation in their bosses. These findings contribute to the training of leaders that foster innovation in organizations and demonstrate the need to continue studying this style of leadership in Colombian companies with regard to innovative behavior among their staff, so as to contribute to the development of organizations more adaptable to the changing circumstances of the environment.

Keywords: leadership; innovation; innovative work behavior; innovational leadership.

\section{Introducción}

Como en otros ámbitos, el contexto educativo requiere de líderes que influyan de forma efectiva sobre sus colaboradores y que estos desarrollen la capacidad de comportarse en función de las necesidades de la organización (Issa-Fontalvo, 2017). En este caso específico, una de las necesidades apremiantes es el desarrollo de procesos innovadores, los cuales solo pueden ser generados por los individuos que trabajen en la organización, conozcan el contexto en el que desarrollan sus actividades y que desde todos los niveles propongan la generación de cambios. Para que estos procesos se generen, se considera que los líderes deben ser promotores de una cultura enfocada en el cambio y que con sus prácticas influyan sobre este comportamiento innovador de sus colaboradores. En este sentido, el desarrollo de condiciones que propicien la innovación es fundamental y en sentido amplio se entendería en función de la creación y optimización de procesos, modificación o creación de servicios, generación de soluciones a problemas existentes o nuevos problemas que se puedan presentar (López Ramos, 2015). Es así como el liderazgo puede potencialmente influir sobre los procesos de innovación en las organizaciones, a través del fomento de un comportamiento innovador en sus trabajadores entendido este como la creación intencional, introducción y aplicación de nuevas ideas en el contexto laboral para favorecer el desempeño (Janssen, 2000) y de esta forma contribuir al desarrollo de organizaciones más adaptables a las actuales condiciones del entorno.

Desde esta perspectiva, el liderazgo se convierte en un factor crucial para el desarrollo de la innovación en las organizaciones a través de su influencia en las prácticas de gestión del talento humano (Pons Verdú \& Ramos López, 2012). En las actuales condiciones 
del entorno, caracterizadas por un alto nivel de complejidad, es una función primordial de los líderes desarrollar prácticas de liderazgo que promuevan en sus empleados comportamientos que faciliten la innovación en las organizaciones. Estas prácticas deben fomentar la construcción de confianza para asumir el riesgo que generan los cambios y estructurar procesos motivacionales que les permita a los colaboradores encontrar alternativas diferentes a problemas cotidianos o complejos del contexto (Contreras et al., 2017). Tales cambios podrán incrementar no solo la calidad de los procesos administrativos sino la efectividad de la gestión en las organizaciones. En este sentido, López Ramos (2015) manifiesta que con sus prácticas, el líder también puede inhibir estos procesos y convertirse en un obstáculo para la innovación, entre ellas señala una supervisión excesiva y escasa tolerancia al error. Estas prácticas pueden potencialmente entorpecer procesos de creación por parte de los empleados, inhibiendo la generación de alternativas novedosas para la resolución de problemas o la mejora de procesos.

Así, cuando se habla de innovación se habla de cambio y del riesgo que este implica, para lo cual los líderes son indispensables. Debido a la necesidad de trabajadores innovadores en las organizaciones y la necesidad de contar con un estilo de liderazgo que lo fomente, Contreras et al. (2019) propusieron una escala para evaluar lo que ellos llamaron liderazgo para la innovación o Innovational Leadership. En este estudio se observó si este tipo de liderazgo guardaba relación con el comportamiento innovador de un grupo de trabajadores del área administrativa de un centro educativo.

Es de resaltar que varios modelos de liderazgo propuestos en las últimas décadas han mostrado cierta preocupación hacia el desarrollo de estos procesos innovadores (López Ramos, 2015). En este sentido, DuBrin (2012) señala que el liderazgo trasformacional por ejemplo, puede fomentar el comportamiento innovador de los trabajadores debido a su capacidad de fortalecer en ellos el desarrollo la autoconfianza y el aprendizaje de manera individual y organizacional. Por otra parte, esta orientación hacia la innovación y el cambio se observa también en la tendencia a vincular el liderazgo con procesos como sostenibilidad y gestión de la diversidad para el cambio innovador. En este sentido, algunos autores señalan la necesidad de generar procesos para lograr organizaciones sostenibles a través de la sinergia que logren desarrollar los lideres entre la diversidad de potencialidades de sus colaboradores (Fernandez, Cho, \& Perry, 2010; Weiss \& Molinaro, 2006).

\section{Liderazgo y su relación con comportamientos innovadores}

Recientemente el estudio del liderazgo en relación con la generación de procesos que promueven la innovación se ha ido incrementando. Con base en un estudio realizado por Contreras et al. (2017) se comenzó a identificar qué tipo de prácticas de liderazgo promueven el comportamiento innovador del trabajador, configurando lo que ellos llamaron "Liderazgo para la Innovación" o innovational leadership un estilo de liderazgo que promueve ese comportamiento en los trabajadores. De acuerdo con el citado estudio, las prácticas de liderazgo pueden influir en el comportamiento innovador de los trabajadores.

No obstante, estudios previos con modelos tradicionales de liderazgo han mostrado su relación con la innovación individual o también llamado, comportamiento innovador del trabajador. Se ha encontrado por ejemplo, que un componente importante del liderazgo transformacional es la construcción de confianza que aporta a que los líderes construyan redes que faciliten la gestión, mejoren la comunicación, compartan puntos de vista y construyan ideas, lo que incluye la motivación inspiradora, la estimulación intelectual y la generación del compromiso, características propias de este estilo de liderazgo (Jung, Wu, \& Chow, 2008). De esta forma, señalan Jung et al. (2008) que se trata de un estilo que impulsa a los empleados a alcanzar los objetivos establecidos, asumir retos, absorber conocimiento; con el cual pueden generar ideas nuevas y compartir valores de manera responsable, lo cual podría fomentar el comportamiento innovador de los empleados. Cabe aclarar que a pesar de que las características anteriores parecen indispensables para generar o promover comportamientos innovadores en los trabajadores, los resultados de los estudios no han sido aún concluyentes. 
Respecto al liderazgo transaccional, cuya característica fundamental es la recompensa contingente (es decir, los líderes premian al trabajador de acuerdo con su desempeño), se ha encontrado que en ocasiones este estilo puede inhibir el comportamiento innovador de los trabajadores debido a que el líder direcciona de manera controlada las acciones a realizar, hace seguimientos cuidadosos de acuerdo con esto, y tiende a evitar las ideas nuevas (Pieterse, Van Knippenberg, Schippers, \& Stam, 2010). Este estilo fomenta que los empleados tiendan a permanecer y mantener determinados patrones de comportamiento y posiblemente no cuentan con un entorno en el que se puedan generar alternativas diferentes. Chen y Chen, (2007) proponen que la combinación de los dos estilos de liderazgo, es decir, tanto el trasformacional como el transaccional, dependiendo de la situación y el contexto pueden generar un resultado favorable para incentivar el comportamiento innovador en los empleados.

Recientemente se menciona el modelo Kotze (Stuart-Kotze \& Dunn, 2008), el cual identifica "comportamientos aceleradores", responsables del crecimiento y del cambio y "comportamientos sostenedores", que son responsables del mantenimiento de la organización. No obstante, los líderes deben desarrollar acciones cotidianas para lo cual el liderazgo transaccional puede ser útil, apalancando comportamientos sostenedores y movilizando la organización hacia el crecimiento y cambio por medio de la función del liderazgo transformacional. López Ramos, (2015) señala que el modelo de Kotze involucra también comportamientos bloqueadores de los directivos como son la presión, la desmotivación y el estrés, entre otros, que pueden generar errores y que posteriormente generan temor en los colaboradores al proponer cambios.

Aún no hay suficiente conocimiento respecto a qué tipo de prácticas de liderazgo promueven la innovación, entendida ésta como un esfuerzo por crear cambios útiles centrados en el potencial económico o social de la organización (Drucker, 1988). Tales cambios incluyen la percepción de lo nuevo, la generación de cambios en diferentes aspectos, en la forma de operar de la organización, en el portafolio de servicios y productos (Van de Ven, 1986).

\section{Comportamiento innovador en el trabajo}

El comportamiento innovador fue inicialmente definido como la fusión entre la generación, promoción y realización de ideas (Scott \& Bruce, 1994). Incluso fue visto como una alternativa de los trabajadores para lidiar con altas cargas de trabajo (Bunce \& West, 1994). Recientemente, el comportamiento innovador se definió como la creación intencional, introducción y aplicación de nuevas ideas dentro de un rol de trabajo, grupo u organización, con el objetivo de beneficiar el desempeño del rol, el grupo o la organización (Janssen, 2000).

El comportamiento innovador se puede definir como la acción de un empleado dirigida a la generación, aplicación e implementación de nuevas ideas, productos, procesos y métodos en su puesto de trabajo, la unidad de departamento u organización, mostrando conceptos como los siguientes: el comportamiento innovador surge como factor de éxito para la supervivencia, generación de valor, competitividad y productividad en las organizaciones (George \& Zhou, 2002). Recientemente, el concepto ha tenido algunas variaciones. Así, De Jong y Den Hartog (2010), afirman que el comportamiento innovador es un proceso compuesto por cuatro dimensiones: la exploración, la generación, la promoción, y la aplicación de las ideas. Por otro lado, De Spiegelaere et al. (2014) afirman que el "comportamiento de los empleados orientados a la generación, introducción y aplicación (en un papel, grupo u organización) de ideas, procesos, productos o procedimientos nuevos, y destinados a beneficiar a la adopción relevante de innovación" (p.144).

En suma, se evidencia que la innovación en una organización muy probablemente depende de la capacidad de innovación tanto de los líderes como de sus empleados, para generar alternativas que al final puedan reflejarse como ventajas competitivas. Por esto, el líder debe propiciar entornos adecuados para el desarrollo, donde se perciba seguridad y confianza, para que los equipos de trabajo puedan usar su creatividad e iniciativa, factores fundamentales para la innovación. Así, teniendo en cuenta la evidencia sobre el efecto del liderazgo en el comportamiento innovador, el propósito de este estudio 
fue identificar el nivel de desarrollo de liderazgo para la innovación que percibe un grupo de empleados y estimar su relación con el comportamiento innovador. De ser así, los resultados de este estudio podrían constituir un insumo importante para el desarrollo de programas que fomenten la gestión a partir de un liderazgo que promueva el comportamiento innovador de los trabajadores.

\section{Método}

Este fue un estudio de tipo descriptivo, de comparación entre grupos en el que se describe como se manifiesta el comportamiento innovador de un grupo de trabajadores administrativos y se observó si los grupos diferían significativamente en la percepción del liderazgo para la innovación de sus jefes inmediatos.

\section{Participantes}

En este estudio participaron 145 trabajadores del área administrativa de una institución privada de educación superior que opera en Bogotá, Colombia. Los participantes constituyeron el 56\% del total de trabajadores del área administrativa incluida en el estudio. Algo más de la mitad de la muestra estuvo compuesta por mujeres (57.20\%). En la tabla 1 pueden observarse las características demográficas de la muestra.

\section{Instrumentos}

Para el desarrollo del estudio, se utilizó la escala de Liderazgo para la innovación (Innovational Leadership Scale, [LL-15]) de Contreras et al. (2019). Esta escala unidimensional está compuesta por 15 ítems con opción de respuesta tipo Likert, cuyo objetivo es identificar el nivel de liderazgo para la innovación que perciben los trabajadores respecto a su jefe inmediato. Se utilizó también la Escala de Comportamiento Innovador en el Trabajo (Innovative work behavior [IwB]) de Janssen (2000).
Esta escala unidimensional consta de 9 ítems, con opción de respuesta tipo Likert, para evaluar tres aspectos del comportamiento innovador; generación, promoción e implementación de ideas. En este estudio los instrumentos mostraron una adecuada confiabilidad, tanto para la Escala de Liderazgo para la Innovación (alfa de Cronbach de 0.95) como para la Escala Comportamiento Innovador en el Trabajo (alfa de Cronbach 0.94).

\section{Procedimiento}

Para la realización de este estudio se obtuvo en primer lugar la autorización de las directivas de la institución a quienes se les ofreció un análisis global de los resultados de su departamento. Posteriormente y previo al diligenciamiento de los instrumentos se obtuvo el consentimiento informado de los trabajadores en el que se especificaba el carácter voluntario de su participación, y el anonimato de sus respuestas. Se aclaró además que tenían libertad de retirarse del estudio en cualquier momento si así lo consideraban. No se les ofreció ningún tipo de retribución por su participación. Una vez obtenido dicho consentimiento se procedió a la aplicación de los instrumentos en línea por medio de la plataforma Limesurvey. Finalmente se procedió a realizar los análisis estadísticos cuyos resultados se presentan a continuación.

\section{Resultados}

La muestra estuvo compuesta por 145 empleados de un área administrativa de la institución de educación superior, ubicada en la ciudad de Bogotá, Colombia. El 57.20\% de los participantes fueron mujeres en su mayoría con edades entre los 30 y 49 años $(77.20 \%)$ y con estudios de posgrado (59.30\%). Se trata de un grupo que en su mayoría no tenía personal a cargo (70.30\%), y llevan trabajando en la institución entre 1 y 10 años (65.50\%). Una alta proporción de la muestra lleva trabajando con su jefe actual entre 1 y 5 años (76.60\%). En la tabla 1 se describe la muestra. 
Tabla 1.

Información descriptiva sobre los participantes

\begin{tabular}{|c|c|c|c|c|c|}
\hline Sexo & $f$ & $\%$ & Personal a cargo & $f$ & $\%$ \\
\hline Hombre & 62 & 42.80 & No & 102 & 70.30 \\
\hline Mujer & 83 & 57.20 & $\mathrm{Si}$ & 43 & 29.70 \\
\hline Rango de edad & & & Antigüedad en la Universidad & & \\
\hline Entre 18 y 29 años & 19 & 13.10 & Menos de 1 año & 7 & 4.80 \\
\hline Entre 30 y 39 años & 77 & 53.10 & Entre 1 y 5 años & 57 & 39.30 \\
\hline Entre 40 y 49 años & 35 & 24.10 & Entre 5 y 10 años & 38 & 26.20 \\
\hline Entre 50 y 59 años & 12 & 8.30 & Entre 10 y 15 años & 26 & 17.90 \\
\hline 60 años o más & 2 & 1.40 & Más de 15 años & 17 & 11.70 \\
\hline Último nivel de formación & & & Antigüedad en cargo actual & & \\
\hline Bachillerato & 1 & 0.70 & Menos de 1 año & 15 & 10.30 \\
\hline Técnica/Tecnológica & 26 & 17.90 & Entre 1 y 5 años & 88 & 60.70 \\
\hline Profesional & 32 & 22.10 & Entre 5 y 10 años & 25 & 17.20 \\
\hline Especialización & 67 & 46.20 & Entre 10 y 15 años & 13 & 9.00 \\
\hline \multirow[t]{5}{*}{ Maestría } & 19 & 13.10 & Más de 15 años & 4 & 2.80 \\
\hline & & & Antigüedad con jefe directo & & \\
\hline & & & Menos de 1 año & 19 & 13.10 \\
\hline & & & Entre 1 y 5 años & 111 & 76.60 \\
\hline & & & Entre 5 y 10 años & 15 & 10.30 \\
\hline
\end{tabular}

Fuente: elaboración propia.

El liderazgo para la innovación y el comportamiento innovador en el trabajo fueron las variables examinadas en este estudio. Los estadísticos descriptivos de las puntuaciones de los participantes en estas variables se presentan en la Tabla 2 junto con el nivel de confiabilidad de los cuestionarios utilizados, los cuales mostraron altos niveles de consistencia interna. En ambas variables se identificaron promedios elevados, indicando la percepción de niveles moderados y altos del liderazgo para la innovación en sus jefes directos, a la vez que reportan niveles altos y moderados de comportamiento innovador en su trabajo.

Tabla 2.

Liderazgo para la innovación y comportamiento innovador en el trabajo de los participantes.

\begin{tabular}{lcccccc}
\hline Variables & $\mathrm{n}$ & Mínimo & Máximo & Media & $\begin{array}{c}\text { Desviación } \\
\text { Típica }\end{array}$ & $\begin{array}{c}\text { Alfa de } \\
\text { Cronbach }\end{array}$ \\
\hline $\begin{array}{l}\text { Liderazgo para la Innovación } \\
\begin{array}{l}\text { Comportamiento Innovador } \\
\text { en el Trabajo }\end{array}\end{array}$ & 145 & 0 & 100 & 73.62 & 19.31 & 0.95 \\
\hline
\end{tabular}

Fuente: elaboración propia. 
Luego se procedió a identificar si el nivel de liderazgo para la innovación que perciben los trabajadores en sus jefes estaba relacionado con el comportamiento innovador que él reporta. Para esto se dividió el nivel de liderazgo en tres grupos siguiendo las recomendaciones de los autores del instrumento (Contreras, et al., 2019): bajo (puntuaciones por debajo de 50), medio (puntuaciones entre 51 y 89 puntos) y alto (puntuaciones entre 90 y 100). En cada grupo se identificó la cantidad de colaboradores y el promedio del comportamiento innovador (Tabla 3).

Tabla 3.

Comportamiento innovador en el trabajo por niveles de liderazgo para la innovación en el grupo de participantes.

\begin{tabular}{lccccc}
\hline Liderazgo para innovación & $n$ & Mínimo & Máximo & Media & Desviación Típica \\
\hline Bajo & 15 & 22 & 100 & 64.07 & 22.53 \\
Medio & 93 & 31 & 100 & 72.58 & 16.03 \\
Alto & 37 & 42 & 100 & 78.60 & 18.24 \\
\hline Total & 145 & 22 & 100 & 73.24 & 17.69 \\
\hline
\end{tabular}

Fuente: elaboración propia.

Las diferencias observadas entre los grupos de colaboradores en función del liderazgo para la innovación se examinaron con un análisis de varianza que resultó significativo $\left(F_{(2,142)}=3.93 ; p=.02\right)$, lo que sugiere que los colaboradores que perciben mayor liderazgo para la innovación en sus jefes, a su vez desarrollan mayor comportamiento innovador en su trabajo. Estas diferencias son aún mayores entre los colaboradores que reportan un bajo liderazgo para la innovación en sus jefes en comparación con los colaboradores que reportan un alto nivel de este estilo de liderazgo (Tabla 4).

Tabla 4.

Comparaciones múltiples (post-hoc) del comportamiento innovador en el trabajo para distintos niveles de liderazgo para la innovación.

\begin{tabular}{|c|c|c|c|c|c|c|}
\hline \multirow{2}{*}{$\begin{array}{l}\text { Liderazgo para la } \\
\text { Innovación (I) }\end{array}$} & \multirow{2}{*}{$\begin{array}{l}\text { Liderazgo para la } \\
\text { Innovación }(\mathrm{J})\end{array}$} & \multirow{2}{*}{$\begin{array}{c}\text { Diferencia } \\
\text { de medias } \\
(\mathrm{I}-\mathrm{J})\end{array}$} & \multirow{2}{*}{$\begin{array}{l}\text { Desv. } \\
\text { Error }\end{array}$} & \multirow{2}{*}{ Sig. } & \multicolumn{2}{|c|}{ Intervalo de confianza al 95\% } \\
\hline & & & & & Límite inferior & Límite superior \\
\hline \multirow{2}{*}{ Bajo } & Medio & -8.50 & 4.82 & 0.21 & -20.45 & 3.44 \\
\hline & Alto & -14.53 & 5.31 & 0.02 & -27.67 & -1.39 \\
\hline \multirow{2}{*}{ Medio } & Bajo & 8.50 & 4.82 & 0.21 & -3.44 & 20.45 \\
\hline & Alto & -6.02 & 3.37 & 0.20 & -14.36 & 2.32 \\
\hline \multirow{2}{*}{ Alto } & Bajo & 14.53 & 5.31 & 0.02 & 1.39 & 27.67 \\
\hline & Medio & 6.02 & 3.37 & 0.20 & -2.32 & 14.36 \\
\hline
\end{tabular}

Nota: Prueba post hoc Scheffe. Diferencias de medias significativas en el nivel .05

Fuente: elaboración propia. 
Finalmente, tanto el liderazgo para la innovación percibido por los colaboradores en sus jefes, como su propio comportamiento innovador en el trabajo fueron examinados por separado en función del contar o no con personal a cargo, a fin de explorar si la relación entre las variables se mantenía bajo estas condiciones. La Tabla 5 muestra las puntuaciones obtenidas; se evidencia que los empleados que tienen personal a cargo obtienen una media de 76 frente a los que no tienen personal a cargo con una media de 72. La diferencia entre los puntajes obtenidos no es estadísticamente significativa (ver tabla 5). Este resultado indica que tener o no perso- nal a cargo no incide en la percepción del liderazgo innovador del jefe inmediato. Así, se sugiere que la percepción de este estilo de liderazgo no depende de la experiencia personal como líder.

En contraste con lo anterior, las puntuaciones obtenidas en el comportamiento innovador en el trabajo indican una diferencia significativa entre los colaboradores con personal a cargo y sin esta responsabilidad. Por lo anterior, podemos decir que quienes tienen personal a cargo reportan consistentemente mayor comportamiento innovador en el trabajo en comparación con los colaboradores sin personal a cargo.

Tabla 5.

Liderazgo para la Innovación y Comportamiento Innovador en el Trabajo clasificado por rol laboral con o sin personal a cargo.

\begin{tabular}{|c|c|c|c|c|c|c|}
\hline & $\begin{array}{c}\text { Personal a } \\
\text { cargo }\end{array}$ & $n$ & Media & Desviación Típica & $t$ & sig \\
\hline \multirow{2}{*}{ Liderazgo para la Innovación } & $\mathrm{Si}$ & 43 & 76.51 & 14.39 & \multirow{2}{*}{1.36} & \multirow{2}{*}{0.17} \\
\hline & No & 102 & 72.40 & 20.98 & & \\
\hline \multirow{2}{*}{$\begin{array}{l}\text { Comportamiento Innovador en el } \\
\text { Trabajo }\end{array}$} & $\mathrm{Si}$ & 43 & 78.49 & 13.36 & \multirow{2}{*}{2.70} & \multirow{2}{*}{0.01} \\
\hline & No & 102 & 71.02 & 18.86 & & \\
\hline
\end{tabular}

Fuente: elaboración propia.

\section{Discusión}

Los resultados muestran que el liderazgo para la innovación se relaciona con el comportamiento innovador de los empleados; lo anterior alineado con lo sugerido en el estudio desarrollado por Contreras et al. (2017) en donde se observó que el liderazgo se relacionaba con el nivel del comportamiento innovador de los trabajadores.

Para las instituciones de educación superior el contar con líderes que propicien comportamientos innovadores en sus empleados puede desarrollar factores de éxito a mediano y largo plazo, dado que su fuerza laboral, al proponer diferentes ideas, impactarán la productividad de manera positiva en sus áreas, como lo mencionan Issa-Fontalvo, (2017), creando y optimizando procesos que resuelvan problemas existentes en el ámbito organizacional, ge- nerando valor a la institución y que al fin de cuentas redunde en la competitividad, desarrollando herramientas nuevas que aporten a la consecución de los objetivos establecidos y el cumplimiento de la misión y visión de la institución.

Como lo manifestó Fernandez, Cho y Perry (2010), si la institución trabaja en pro de la orientación al logro, las relaciones positivas, el cambio organizacional, entre otras, facilitará que dichas características sean apropiadas por sus líderes y en consecuencia, faciliten el desarrollo del comportamiento innovador en sus empleados.

Los resultados obtenidos en este estudio claramente respaldan la posición asumida por Contreras et al., (2017) quienes manifiestan que el liderazgo transaccional y transformacional pueden ejercer un efecto sobre el comportamiento innovador, aunque de 
forma distinta. Así, puede afirmarse que el liderazgo impacta de manera importante el desarrollo de comportamientos innovadores en los empleados. Tal como afirmó DuBrin, (2012), el desarrollo en los empleados de comportamientos innovadores aunado a factores de motivación y autoconfianza, facilitarán que se den comportamientos que aporten a la innovación, satisfaciendo su interés, aprendiendo de manera individual y como equipo de trabajo, enfocando dichos aprendizajes en pro de la organización.

Se considera importante tener en cuenta que el liderazgo, como lo manifiestan Jung et al. (2008), utilizado para el fortalecimiento de la confianza en los empleados, facilitará el desarrollo de la comunicación asertiva y el respeto por las ideas en los demás, permitiendo que se manifiesten comportamientos innovadores que propicien en los empleados la construcción de alternativas innovadoras conjuntas, que impacten procesos trasversales y que aporten a la competitividad organizacional. Adicionalmente se puede observar que los resultados encontrados pueden llegar a confirmar lo manifestado por Pieterse et al. (2010), mostrando en alguna medida que las prácticas de liderazgo efectivamente influyen en el comportamiento innovador de los colaboradores.

Este estudio provee aportes en cuanto a la gestión del campo organizacional, se debe seguir estudiando las prácticas de liderazgo en relación con el comportamiento innovador a fin de desarrollar conocimientos que impacten a las organizaciones, haciéndolas más flexibles a las circunstancias cambiantes del entorno. El instrumento desarrollado para estimar el liderazgo para la innovación ha demostrado buen nivel de fiabilidad, se recomienda su uso con el fin de verificar su validez y confiabilidad en distintos contextos organizacionales.

\section{Implicaciones prácticas para la gestión}

Los resultados obtenidos permiten verificar la existencia de un liderazgo para la innovación y sustentar la relación entre liderazgo y comportamiento innovador de los trabajadores. Así mismo, es importante crear mayor conciencia en las organizaciones frente al importante rol que tienen los líderes en el desarrollo de comportamientos innovadores en sus colaboradores, motivándolos, generándoles autonomía, compromiso, permitiendo la construcción de ideas de manera individual y conjunta, orientándolos al resultado, fomentando la creatividad, y con todo esto contribuir al cumplimiento de los objetivos organizacionales estructurados.

Por lo anterior y teniendo cuenta la importancia de los líderes en la organización sería interesante reconocer que proceso de formación de los mismos es indispensable para aportar al desarrollo de habilidades interpersonales y técnicas que propicien el fortalecimiento del comportamiento innovador de sus empleados.

De este estudio puede inferirse que las organizaciones que quieran desarrollar un liderazgo para la innovación deben considerar procesos que promuevan la formación en habilidades de liderazgo como comunicación, orientación al logro, gestión y adaptación al cambio, entre otras. Así como habilidades técnicas, todo esto fundamentado en la confianza y la promoción del cambio a través de sus empleados (Jung et al., 2008).

Actividades tales como la creación de espacios de construcción de ideas innovadoras de manera individual, grupal y por equipos de trabajo de todos los niveles, pueden fomentar el comportamiento innovador. Estructurar soluciones a problemas, implementando metodologías novedosas que aporten a su gestión y seguimiento del cambio puede promover en los miembros de la organización el desarrollo de hábitos de pensamiento diferente para solucionar los obstáculos cotidianos. De esta manera es posible que se fomente la creatividad, se genere el empoderamiento y la capacidad de gestión, habilidades directamente relacionadas con la innovación organizacional (López-Ramos, 2015).

Se recomienda también, socializar los resultados positivos obtenidos por los logros alcanzados y los resultados no favorables para no repetirlos, generar alternativas de éxito en futuros proyectos, reconociendo a los autores de dichas ideas, generando motivación para aumentar estos comportamientos deseados. Es importante mencionar que el desarrollo de comportamientos que van cambiando la 
cultura organizacional, es una labor a largo plazo, por lo tanto, los procesos de formación deben ser constantes, oportunos y con el seguimiento adecuado para consolidar una cultura de la innovación en donde los lideres logren promover el comportamiento innovador de sus trabajadores.

\section{Referencias}

Bunce, D. \& West, M. (1994). Changing work environments: Innovative coping responses to occupational stress. Work \& Stress, 8(4), 319-331.

Chen, J. \& Chen, I. (2007). The relationships between personal traits, leadership styles, and innovative operation. Proceedings of the 13th Asia Pacific Management Conference, Melbourne, Australia, 420-425.

Contreras, F., Espinosa, J. C., Dornberger, U., \& Cuero Acosta, Y. A. (2017). Leadership and Employees' Innovative Work Behavior: Test of a Mediation and Moderation Model. Asian Social Science, 13(9), 9-25. https://doi.org/10.5539/ ass.v13n9p9

Contreras, F., Espinosa, J.C., Dornberger, U. (2019). Innovational leadership: Design of a scale to asses this new model. Manuscrito en preparación.

De Jong, J. \& Den Hartog, D. (2010). Measuring innovative work behaviour. Creativity and innovation management, 19(1), 23-36.

De Spiegelaere, S., Van Gyes, G., De Witte, H., Niesen, W., \& Van Hootegem, G. (2014). On the relation of job insecurity, job autonomy, innovative work behaviour and the mediating effect of work engagement. Creativity and innovation management, 23(3), 318-330.

Drucker, P. F. (1988, enero 1). The Coming of the New Organization. Harvard Business Review, (January 1988). Recuperado de https://hbr.org/1988/01/ the-coming-of-the-new-organization

DuBrin, A. J. (2012). Leadership: Research Findings, Practice, and Skills (Edición: 7). Australia; Mason, он: Cengage Learning.
Fernandez, S., Cho, Y. J., \& Perry, J. L. (2010). Exploring the link between integrated leadership and public sector performance. The Leadership Quarterly, 21(2), 308-323. https:// doi.org/10.1016/j.leaqua.2010.01.009

George, J. M. \& Zhou, J. (2002). Understanding when bad moods foster creativity and good ones don't: The role of context and clarity of feelings. Journal of Applied Psychology, 87(4), 687-697. https://doi.org/10.1037/0021-9010.87.4.687

Issa-Fontalvo, S. M. (2017). Habilidades del liderazgo para una cultura de innovación en la gerencia de las universidades del distrito de Santa Marta. Revista Academia y Virtualidad, 10(1), 56-67. https://doi.org/10.18359/ravi.2685

Janssen, O. (2000). Job demands, perceptions of effort-reward fairness and innovative work behaviour. Journal of Occupational and Organizational Psychology, 73(3), 287-302. https://doi.org/10.1348/096317900167038

Jung, D. D., Wu, A., \& Chow, C. W. (2008). Towards understanding the direct and indirect effects of CEOS' transformational leadership on firm innovation. The leadership quarterly, 19(5), 582-594.

López Ramos, A. (2015). Diseño metodológico para la creación y desarrollo de organizaciones innovadoras, basadas en un modelo de liderazgo que genere entornos facilitadores de la creatividad, implantación de procesos de innovación y creación de productos y servicios innovadores (Phd, E.T.S.I. Industriales (UPM)). Recuperado de http://oa.upm.es/37212/

Pieterse, A. N., Van Knippenberg, D., Schippers, M., \& Stam, D. (2010). Transformational and transactional leadership and innovative behavior: The moderating role of psychological empowerment. Journal of organizational behavior, 31(4), 609-623.

Pons Verdú, F. J. \& Ramos López, J. (2012). Influencia de los estilos de liderazgo y las prácticas de gestión de RRHH sobre el clima organizacional de innovación. Revista de Psicología del 
Trabajo y de las Organizaciones, 28(2), 81-98. https://doi.org/10.5093/tr2012a7

Scott, S. G. \& Bruce, R. A. (1994). Determinants of innovative behavior: A path model of individual innovation in the workplace. Academy of management journal, 37(3), 580-607.

Stuart-Kotze, R. \& Dunn, C. (2008). Who Are Your Best People?: How to find, measure and manage your top talent. London: Pearson education.
Van de Ven, A. H. (1986). Central Problems in the Management of Innovation. Management Science, 32(5), 590-607.

Weiss, D. \& Molinaro, V. (2006). Integrated leadership development. Industrial and Commercial Training, 38(1), 3-11. https://doi. org/10.1108/00197850610700763 